\title{
CHANGES IN mRNA EXPRESSION OF MEMBERS OF TGFB1-ASSOCIATED PATHWAYS IN HUMAN LEUKOCYTES DURING EBV INFECTION
}

\author{
Elena Nikolaevna Filatova*, Nikolay AleKsandrovich SaKharnov, \\ DMITRY IGOREVICH KNYAZEV and OLEG VLADIMIROVICH UTKIN
}

Laboratory of Molecular Biology and Biotechnology, Blokhina Scientific Research Institute of Epidemiology and Microbiology of Nizhny Novgorod, Nizhny Novgorod, Russian Federation

(Received: 5 September 2018; accepted: 24 October 2018)

\begin{abstract}
Transforming growth factor $\beta 1$ (TGFB1) likely contributes to the pathogenesis of Epstein-Barr virus (EBV)-mediated cancer. A microarray containing 59 probes for detecting mRNA of members of TGFB1-associated pathways was developed. mRNA expression of TGFB1 receptors and members of connected pathways were examined in peripheral blood leukocytes of patients during acute EBV infection and after recovery. $T G F B 1$ and $T G F B R 2 \mathrm{mRNA}$ expression was increased in patients with EBV infection. Similarly, mRNA expression of protein kinase C (PRKCB), MAP3K7, PDLIM7, and other members of TGFB1 and NF- $\mathrm{KB}$ signaling pathways increased. A shift of mRNA transcript variant expression of some key members (TGFBR2, $P R K C B$, and $N F K B I B)$ of involved signaling pathways was detected. After the patients' recovery, most of the altered mRNA expression has been normalized. We speculate that in patients with EBV infection, members of TGFB1-associated pathways contribute to the suppression of proapoptotic and induction of pro-survival factors in leukocytes. The modulation of TGFB1-associated pathways may be considered as a potential risk factor in the development of EBV-associated tumors in patients with acute EBV infection.
\end{abstract}

Keywords: EBV, TGFB1, NF-кB, PRKCB, apoptosis, proliferation, leukocytes

\section{Introduction}

Epstein-Barr virus (EBV, Human gammaherpesvirus 4) is a ubiquitous human oncogenic virus that persists in over $90 \%$ of the world's population. It is known that EBV makes a significant contribution to several human cancers, such as B-cell, T-cell, and NK-cell lymphomas [1].

*Corresponding author; E-mail: filatova@nniiem.ru 
Transforming growth factor $\beta 1$ (TGFB1) is a multifunctional polypeptide that plays a critical role in the regulation of cell proliferation, differentiation, and growth [2]. It induces EBV lytic infection by activating the expression of EBV's latent-lytic switch BZLF1 gene through the canonical SMAD pathway [3]. TGFB1 might provide a significant contribution to the transforming potential of EBV. In patients with EBVassociated nasopharyngeal carcinomas, high serum level of TGFB1 is an unfavorable prognostic [4], for it can enhance tumor progression by stimulating epithelialmesenchymal transition [5]. On the other hand, in EBV-infected gastric epithelial cell lines, TGFB1 mediates growth inhibition and apoptosis [6].

The dual nature of TGFB1 is determined by its ability to crosstalk with MAPK8 and NF-кB signal pathways (hereafter official full names of genes are provided in Supplementary Table I; available at https://cloud.nniiem.ru/s/ 1FdiA0LgZEOrNtV) [7-9]. Regulatory molecules like protein kinase C PRKCB are capable of preventing TGFB1-dependent apoptosis in tumor cells by suppression of activity of transcription modulator SMAD3 [10] and activation of MAPK8 and NF- $\kappa \mathrm{B}$ signaling pathway, thereby promoting cell survival [11-13]. In addition, for some members of TGFB1-associated pathways, multiple mRNA transcript variants with different functions are identified.

All of the above make it difficult to assess the contribution of TGFB1 to the development of EBV-associated pathology. Expression pattern of members of TGFB1-connected pathways in blood cells of patients with non-oncological EBV infection is currently poorly understood. Simultaneously, this knowledge will improve our understanding of the mechanisms of transformation of infected cells and will offer new strategies for the therapy of EBV-associated cancer.

The aim of this study was to examine the expression pattern of members of TGFB1-associated pathways in leukocytes of patients with acute EBV infection.

\section{Materials and Methods}

A microarray design was developed using "Splice variants microarray design pipeline" algorithm [14]. A total of 59 target probes were selected including 23 gene "total mRNA" probes (designed to detect all mRNA transcript variants of one gene) and 36 "mRNA transcript variant" probes (designed to detect one specific mRNA transcript variant of one gene; Supplementary Table I). Seventy "negative control" probes, design based on Rhizobium rubi genome, were included in each microarray. Microarray design was adapted for probe synthesis using Layout Designer software (CustomArray Inc., WA, USA). Probe synthesis was conducted in situ on the $12 \mathrm{~K}$ microarray slides by the B3 Synthesizer (CustomArray Inc.). The synthesis and post-synthetic slide deprotection were 
carried out in accordance with the manufacturer's protocols using recommended reagents.

Blood samples were obtained from 7- to 18-year-old $($ median $=12)$ patients diagnosed with infectious mononucleosis. Acute EBV infection was confirmed by clinical symptoms and laboratory tests (ELISA and PCR). Blood samples were taken in acute phase of infection (EBVinf, $n=6$ ) before the beginning of symptomatic drug therapy, which included, by prescription, ibuprofen, acetaminophen, and xylometazoline, and 2 months after apparent clinical recovery (EBVrec, $n=5$ ). Due to the high similarity of the pathogenesis mechanism and symptoms of the disease, children of 8-17 (median $=11)$ years old with confirmed acute Human herpesvirus 6 infection (HHV-6inf, $n=7$ and HHV-6rec, $n=7$ ) were used as the comparison group. Another group comprised healthy volunteers of 8-17 (median $=11$ ) years old without clinical and laboratory signs of infection (NORM, $n=17$ ). All procedures performed in studies involving human participants were in accordance with the ethical standards of the institutional and national research committee and with the 1964 Helsinki Declaration and its later amendments or comparable ethical standards. Informed consent was obtained from all individual participants' parents or legal guardians included in the study.

Blood samples were treated with a hemolytic solution (CRIE, Russia) to remove red blood cells. From the obtained leukocyte fraction, total RNA was isolated and proceeded to biotin-labeled antisense RNA according to common protocols using commercial kits and reagents (Evrogen, Russia; DNA synthesis, Russia; Thermo Scientific, EU). Hybridization of the labeled RNA on the microchip and measurement of the amperometric signal were carried out using a commercial set of ElectraSense reagents and equipment (CustomArray Inc.), according to the manufacturer's recommendations.

All further calculations were performed using $\mathrm{R}$ software (RStudio version 1.1.453, RStudio, Inc., Boston, MA, USA). Data were normalized using the subset quantile normalization based on the non-specific control features [15]. To estimate fold change, sample means of NORM and EBV were calculated for each target probe. Fold change $(\%)$ was calculated as [(EBV sample mean $\times 100 /$ NORM sample mean) - 100]. For each target probe, NORM sample versus EBVinf sample were tested using $t$-test; $p=0.05$ was selected as threshold value. The same procedures were performed using EBVrec, HHV-6inf, and HHV-6rec samples.

\section{Results}

It was shown that EBV infection was followed by change of expression pattern of TGFB1 and TGFB1-connected pathways in human leukocytes 


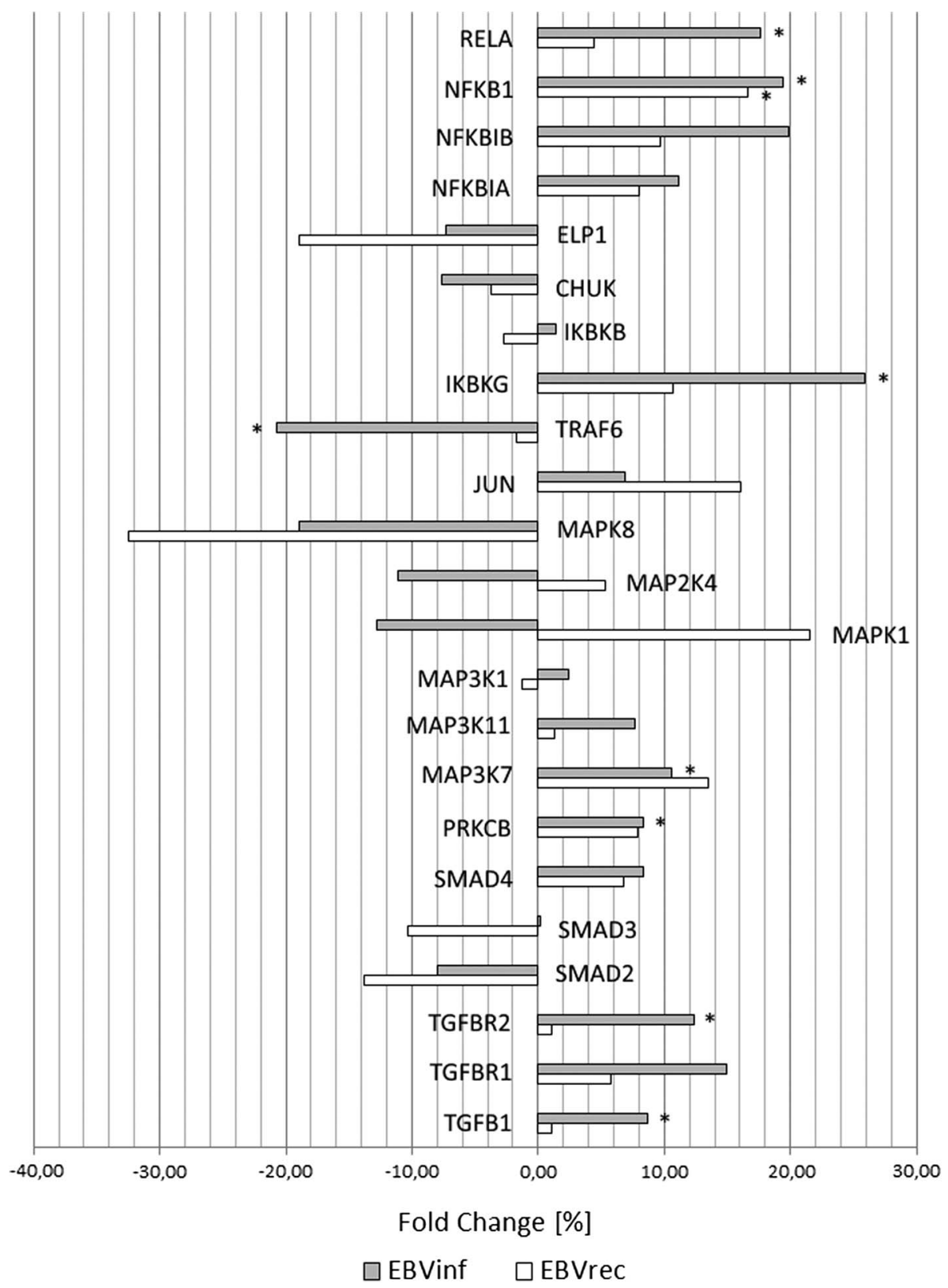

Figure 1. Fold change of total mRNA expression of members of TGFB1-connected pathways in leukocytes of patients with acute $\mathrm{EBV}$ infection $(\mathrm{EBV})$ and after apparent clinical recovery (EBVrec) in comparison with healthy volunteers. *Difference is statistically significant $(p<0.05)$. For mRNA transcript variants, see Supplementary Table I 
(Figure 1, Supplementary Table I, available at https:/cloud.nniiem.ru/s/ 1FdiA0LgZEOrNtV). TGFB1 total mRNA expression and mRNA transcript variant 1 expression increased in EBVinf compared with NORM. After the recovery, $T G F B 1$ expression has been normalized.

Expression of some of the members of SMAD-mediated TGFB1 signaling pathway was increased in EBVinf compared with healthy volunteers (NORM). In EBVinf leukocytes TGFBR2 total mRNA expression and mRNA transcript variant 2 expression, $S M A D 2$ mRNA transcript variant 2 expression increased. TGFBR1, $S M A D 3$, and SMAD4 mRNA expression did not change in EBVinf compared with NORM. In EBVrec, expression of mRNA of members of SMAD-mediated TGFB1 signaling pathway did not differ compared with NORM, except for SMAD2 mRNA transcript variant 2 expression, which remained elevated. In EBVinf, $P R K C B$ total mRNA expression and mRNA transcript variant 2 expression decreased compared with NORM. Apparent clinical recovery was accompanied by normalization of $P R K C B$ mRNA expression.

In EBVinf, MAP $3 K 7$ total mRNA expression increased compared with NORM. This indicator returned to normal after clinical recovery. EBV infection was not accompanied by the change in the mRNA expression of other participants of MAPK8 signaling pathways (MAP3K11, MAP3K1, MAPK1, MAP2K4, $M A P K 8$, and JUN). TRAF6 total mRNA expression and expression of both TRAF6 mRNA transcript variants decreased in EBVinf compared with NORM. After recovery, the TRAF6 mRNA expression did not differ from NORM.

mRNA expression of some members of NF- $\mathrm{KB}$ signaling pathway was modulated in leukocytes of patients with EBV infection. Among members of IKK complex, CHUK, IKBKB, and ELPI mRNA expression did not change, but $I K B K G$ total mRNA expression and mRNA transcript variants 3 and 4 expression increased in EBVinf compared with NORM. After clinical recovery, IKBKG mRNA transcript variant 4 expression remained elevated. Among NF- $\kappa B$ inhibitors, NFKBIA mRNA expression did not change in patients with EBV. $N F K B I B$ total mRNA expression also did not change in EBVinf compared with NORM, but $N F K B I B$ mRNA transcript variants 1 and 2 expression increased, whereas $N F K B I B$ non-coding mRNA transcript variant 3 expression decreased. $N F K B I B$ mRNA transcript variants 1 and 2 expression remained elevated in EBVrec, and $N F K B I B$ non-coding mRNA transcript variant expression normalized after recovery. Among NF- $\mathrm{KB}$ subunits, $N F K B 1$ total mRNA expression, $N F K B 1$ mRNA transcript variant 1 expression, and RELA total mRNA expression increased, whereas $N F K B 1$ mRNA transcript variant 3 expression decreased in EBVinf compared with NORM. NFKB1 total mRNA expression and NFKB1 mRNA transcript variant 1 expression remained elevated after apparent clinical recovery. 
We did not reveal similar mRNA expression pattern in children with acute HHV-6 infection (Supplementary Table I).

\section{Discussion}

We speculate that revealed changes of mRNA expression pattern in leukocytes of patients with EBV were caused by acute EBV infection. Our results also suggest that members of TGFB1-associated pathways play significant role in viral pathogenesis. The revealed changes might be associated with suppression of apoptosis and promotion of leukocytes' proliferation through activation of protein kinase C PRKCB (Figure 2) or/and modulation of mRNA transcript variants expression of some key members (TGFBR2, PRKCB, and NFKBIB) of involved signaling pathways.

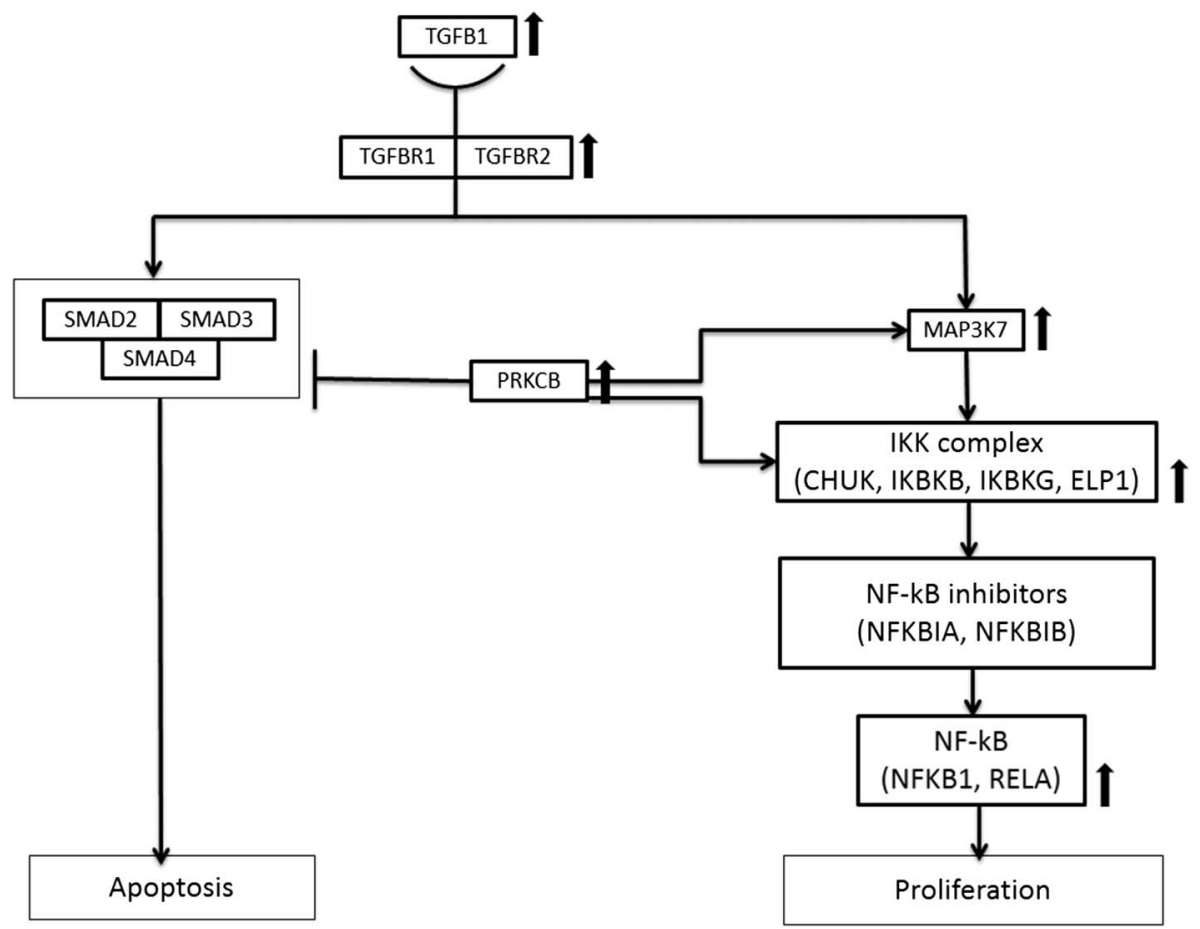

Figure 2. mRNA expression of members of TGFB1-associated pathways in leukocytes of patients with acute EBV infection. Thick arrows show changes in total mRNA expression of some members of TGFB1-associated pathways in infected patients compared with healthy volunteers. Detected mRNA expression pattern's changes might result in inhibiting TGFB1-dependent apoptosis, activation of $\mathrm{NF}-\kappa \mathrm{B}$, and stimulation of cell proliferation 
Specifically, TGFBR2 mRNA transcript variant 2, which was highly expressed in EBVinf leukocytes, is typically detected in leukemia cells where it is associated with impaired cell differentiation and proliferation, as well as suppression of apoptosis [16]. Expression of TGFBR2 mRNA transcript variant 1, which is able to stimulate TGBF1-dependent suppression of proliferation and apoptosis in tumor cell lines [17], did not change in EBVinf. In this study, PRKCB mRNA transcript variant 2 expression decreased in patients with EBV, and similar results were observed in colorectal cancer cells in which $P R K C B$ mRNA transcript variant 2 isozyme reverses cell transformation. In patients with colorectal cancer, low levels of $P R K C B$ mRNA transcript variant 2 expression serve as a predictor for poor survival outcome [18]. In addition, according to our findings, expression of PRKCB mRNA transcript variant 1, which is directly involved in the MAPK and NF- $\mathrm{KB}$ activation and cell proliferation, increased in EBVinf blood, although it was not statistically significant. Again, in EBVinf leukocytes, $N F K B I B$ mRNA expression pattern changed upward, since protein coding mRNA transcript variant expression increased, whereas non-coding transcript variant expression decreased.

All the above contribute to the suppression of proapoptotic and induction of pro-survival factors in leukocytes. The results allow us to consider the modulation of TGFB1-associated pathways as a potential risk factor in the development of $\mathrm{EBV}$-associated tumors in patients with acute EBV infection.

The data sets generated and analyzed during this study are available from the corresponding author on reasonable request.

\section{Conflict of Interest}

No conflict of interest was declared by the authors.

\section{References}

1. Dojcinov, S. D., Fend, F., Quintanilla-Martinez, L.: EBV-positive lymphoproliferations of B- T- and NK-cell derivation in non-immunocompromised hosts. Pathogens 7, 28 (2018).

2. Yan, X., Xiong, X., Chen, Y.-G.: Feedback regulation of TGF- $\beta$ signaling. Acta Biochim Biophys Sin 50, 37-50 (2018).

3. Iempridee, T., Das, S., Xu, I., Mertz, J. E.: Transforming growth factor $\beta$-induced reactivation of Epstein-Barr virus involves multiple Smad-binding elements cooperatively activating expression of the latent-lytic switch $B Z L F 1$ gene. J Virol 85, 7836-7848 (2011).

4. Xu, J., Menezes, J., Prasad, U., Ahmad, A.: Elevated serum levels of transforming growth factor $\beta 1$ in Epstein-Barr virus-associated nasopharyngeal carcinoma patients. Int J Cancer 84, 396-399 (1999). 
5. Xie, L., Law, B. K., Chytil, A. M., Brown, K. A., Aakre, M. E., Moses, H. L.: Activation of the Erk pathway is required for TGF- $\beta 1$-induced EMT in vitro. Neoplasia (New York, NY) 6, 603-610 (2004).

6. Fukuda, M., Kurosaki, W., Yanagihara, K., Kuratsune, H., Sairenji, T.: A mechanism in Epstein-Barr virus oncogenesis: Inhibition of transforming growth factor- $\beta 1$-mediated induction of MAPK/p21 by LMP1. Virology 302, 310-320 (2002).

7. Al-Azayzih, A., Gao, F., Goc, A., Somanath, P. R.: TGF $\beta 1$ induces apoptosis in invasive prostate cancer and bladder cancer cells via Akt-independent, p38 MAPK and JNK/SAPKmediated activation of caspases. Biochem Biophys Res Commun 427, 165-170 (2012).

8. Bailey, K. L., Agarwal, E., Chowdhury, S., Luo, J., Brattain, M. G., Black, J. D., Wang, J.: TGF $\beta / \mathrm{Smad} 3$ regulates proliferation and apoptosis through IRS-1 inhibition in colon cancer cells. PLoS One 12, e0176096 (2017).

9. Freudlsperger, C., Bian, Y., Contag Wise, S., Burnett, J., Coupar, J.: TGF- $\beta$ and NF-kB signal pathway cross-talk is mediated through TAK1 and SMAD7 in a subset of head and neck cancers. Oncogene 32, 1549-1559 (2013).

10. Yakymovych, I., Ten Dijke, P., Heldin, C.H., Souchelnytskyi, S.: Regulation of Smad signaling by protein kinase C. FASEB J 15, 553-555 (2001).

11. Kawakami, T., Kawakami, Y., Kitaura, J.: Protein kinase C beta (PKC beta): Normal functions and diseases. J Biochem (Tokyo) 132, 677-682 (2002).

12. Krappmann, D., Patke, A., Heissmeyer, V., Scheidereit, C.: B-cell receptor- and phorbol ester-induced NF- $\mathrm{kB}$ and c-Jun $\mathrm{N}$-terminal kinase activation in $\mathrm{B}$ cells requires novel protein kinase C's. Mol Cell Biol 21, 6640-6650 (2001).

13. Uemura, N., Kajino, T., Sanjo, H., Sato, S., Akira, S., Matsumoto, K., Ninomiya-Tsuji, J.: Tak1 is a component of the Epstein-Barr virus Lmp1 complex and is essential for activation of JNK but not of NF-кB. J Biol Chem 281, 7863-7872 (2006).

14. Solntsev, L. A., Starikova, V. D., Sakharnov, N. A., Knyazev, D. I., Utkin, O. V.: Strategy of probe selection for studying mRNAs that participate in receptor-mediated apoptosis signaling. Mol Biol 49, 457-465 (2015).

15. Wu, Z., Aryee, M. J.: Subset quantile normalization using negative control features. J Comput Biol 17, 1385-1395 (2010).

16. Wu, Y., Su, M., Zhang, S., Cheng, Y., Liao, X. Y., Lin, B. Y., Chen, Y. Z.: Abnormal expression of TGF-beta type II receptor isoforms contributes to acute myeloid leukemia. Oncotarget 8, 10037-10049 (2016).

17. Wan, J., Sun, L., Mendoza, J. W., Chui, Y. L., Huang, D. P.: Elucidation of the c-Jun $\mathrm{N}$-terminal kinase pathway mediated by Epstein-Barr virus-encoded latent membrane protein 1. Mol Cell Biol 24, 192-199 (2004).

18. Dowling, C. M., Phelan, J., Callender, J. A., Cathcart, M. C., Mehigan, B.: Protein kinase C beta II suppresses colorectal cancer by regulating IGF-1 mediated cell survival. Oncotarget 7, 20919-20933 (2016). 